\title{
On Coupled Common Fixed Point Theorems for Nonlinear Contractions with the Mixed Weakly Monotone Property in Partially Ordered S-Metric Spaces
}

\author{
Mi Zhou ${ }^{1}$ and Xiao-Lan Liu ${ }^{2,3}$ \\ ${ }^{1}$ School of Polytechnics, Sanya University, Sanya, Hainan 572000, China \\ ${ }^{2}$ Department of Mathematics, Sichuan University of Science and Engineering, Zigong, Sichuan 643000, China \\ ${ }^{3}$ Sichuan Province University Key Laboratory of Bridge Non-Destruction Detecting and Engineering Computing,
}

Zigong, Sichuan 643000, China

Correspondence should be addressed to Xiao-Lan Liu; stellalwp@163.com

Received 18 November 2015; Accepted 29 December 2015

Academic Editor: Hugo Leiva

Copyright (C) 2016 M. Zhou and X.-L. Liu. This is an open access article distributed under the Creative Commons Attribution License, which permits unrestricted use, distribution, and reproduction in any medium, provided the original work is properly cited.

The main aim of this paper is to establish some coupled common fixed point theorems under a Geraghty-type contraction using mixed weakly monotone property in partially ordered $S$-metric space. Also, we give some sufficient conditions for the uniqueness of a coupled common fixed point. Some examples are provided to demonstrate the validity of our results.

\section{Introduction and Preliminaries}

One of the most important results in fixed point theory is the Banach Contraction Principle (BCP for short) proposed by Banach [1]. After that, there were many authors who have studied and proved the results for fixed point theory by generalizing the Banach Contraction Principle in several directions. One of the celebrated results was given by Geraghty [2].

For the sake of convenience, we recall Geraghty's theorem. Let $\mathfrak{F}$ be the family of all functions $\beta:[0, \infty) \mapsto[0,1)$ satisfying the condition:

$$
\begin{aligned}
\lim _{n \rightarrow \infty} \beta\left(t_{n}\right) & =1 \Longrightarrow \\
\lim _{n \rightarrow \infty} t_{n} & =0 .
\end{aligned}
$$

Geraghty [2] proved the following unique fixed point theorem in complete metric spaces.
Theorem 1 (see [2]). Let $(X, d)$ be a complete metric space and let $T: X \mapsto X$ be an operator. Suppose that there exists $\beta \in \mathfrak{F}$ such that

$$
d(T x, T y) \leq \beta(d(x, y)) d(x, y),
$$

for all $x, y \in X$. Then $T$ has a unique fixed point $x^{*} \in X$.

Later, Amini-Harandi and Emami [3] generalized this result to the setting of partially ordered metric spaces as follows.

Theorem 2 (see [3]). Let $(X, d)$ be a complete partially ordered metric space and let $f: X \mapsto X$ be an increasing self-mapping such that there exists $x_{0} \in X$ such that $x_{0} \preceq f x_{0}$. Suppose that there exists $\beta \in \mathfrak{F}$ such that

$$
d(f x, f y) \leq \beta(d(x, y)) d(x, y),
$$

for all $x, y \in X$ satisfying $x \leq y$ or $x \geq y$. Then, in each of the following two cases, the mapping $f$ has at least one fixed point in $X$ :

(1) $f$ is continuous or, 
(2) for any nondecreasing sequence $\left\{x_{n}\right\}$ in $X$, if $x_{n} \rightarrow x \in$ $X$ as $n \rightarrow \infty$, then $x_{n} \preceq x$ for all $n \geq 1$.

If, moreover, for all $x, y \in X$, there exists $z \in X$ comparable with $x$ and $y$, then the fixed point of $f$ is unique.

For more generalizations of Theorems 1 and 2, see [4-7].

On the other hand, several authors have studied fixed point theory in generalized metric spaces. For details, we refer readers to [8-13]. In 2012, Sedghi et al. [14] have introduced the notion of an $S$-metric space and proved that this notion is a generalization of a metric space. Also, they have proved some properties of $S$-metric spaces and some fixed point theorems for a self-map on an $S$-metric space. An interesting work is that we can naturally transport certain results in metric spaces and known generalized metric spaces to $S$ metric spaces. After that, Sedghi and Dung [15] proved a general fixed point theorem in $S$-metric spaces which is a generalization of [14, Theorem 3.1] and obtained many analogues of fixed point theorems in metric spaces for $S$ metric spaces. In [16], Gordji et al. have introduced the concept of a mixed weakly monotone pair of maps and proved some coupled common fixed point theorems for contractivetype maps using the mixed weakly monotone property in partially ordered metric spaces. These results are of particular interest to state coupled common fixed point theorems for maps with mixed weakly monotone property in partially ordered $S$-metric spaces. In 2013, Dung [17] used the notion of a mixed weakly monotone pair of maps to state a coupled common fixed point theorem for maps on partially ordered $S$-metric spaces and generalized the main results of [16-18] into the structure of $S$-metric spaces.

In this paper, motivated by the developments discussed above, we state some coupled common fixed point theorems for a pair of mappings with the mixed weakly monotone property satisfying a generalized contraction by using the ideas of Geraghty [2] in partially ordered $S$-metric spaces. Also, we give some sufficient conditions for the uniqueness of a coupled common fixed point. Some examples are provided to illustrate our main theorems.

In the sequel, the letters $\mathbb{R}, \mathbb{R}^{+}$, and $\mathbb{N}$ will denote the set of all real numbers, the set of all nonnegative real numbers, and the set of all positive integers, respectively.

Let $(X, \preccurlyeq)$ be a partially ordered set. Then $X \times X$ is a partially ordered set with partial order $\preccurlyeq$ defined by

$$
\begin{gathered}
(x, y) \preccurlyeq(u, v) \Longleftrightarrow \\
x \preccurlyeq u, v \preccurlyeq y,
\end{gathered}
$$

$\forall x, y, u, v \in X$

Definition 3 ([14, Definition 2.1]). Let $X$ be a nonempty set. An $S$-metric on $X$ is a function $S: X^{3} \mapsto[0, \infty)$ that satisfies the following conditions for all $x, y, z, a \in X$ :

(1) $S(x, y, z)=0$ if and only if $x=y=z$.

(2) $S(x, y, z) \leq S(x, x, a)+S(y, y, a)+S(z, z, a)$.

The pair $(X, S)$ is called an $S$-metric space.
The following is an intuitive geometric example for $S$ metric spaces.

Example 4 ([14, Example 2.4]). Let $X=\mathbb{R}^{2}$ and let $d$ be an ordinary metric on $X$. Put

$$
S(x, y, z)=d(x, y)+d(x, z)+d(y, z)
$$

for all $x, y, z \in \mathbb{R}^{2}$; that is, $S$ is the perimeter of the triangle given by $x, y, z$. Then $S$ is an $S$-metric on $X$.

Lemma 5 ([17, Lemma 1.4]). Let $(X, S)$ be an S-metric space. Then

$$
\begin{aligned}
& S(x, x, z) \leq 2 S(x, x, y)+S(y, y, z), \\
& S(x, x, z) \leq 2 S(x, x, y)+S(z, z, y),
\end{aligned}
$$

for all $x, y, z \in X$.

Lemma 6 ([14, Lemma 2.5]). Let $(X, S)$ be an $S$-metric space. Then $S(x, x, y)=S(y, y, x)$, for all $x, y \in X$.

Lemma 7 (see [16]). Let $(X, d)$ be a metric space. Then $X \times X$ is a metric space with metric $D_{d}$ given by

$$
D_{d}((x, y),(u, v))=d(x, u)+d(y, v),
$$

for all $x, y, u, v \in X$.

Lemma 8. Let $(X, S)$ be an S-metric space. Then $X \times X$ is an $S$-metric space with $S$-metric $D_{s}$ given by

$$
D_{s}((x, y),(u, v),(w, s))=S(x, u, w)+S(y, v, t) \text {, }
$$

for all $x, y, u, v, w, t \in X$.

Proof. For all $x, y, u, v, w, t \in X$, it is obvious that the first condition of $S$-metric for $D_{s}$ holds true.

We only need to check the second condition of $S$-metric:

$$
\begin{aligned}
D_{s}( & (x, y),(u, v),(w, t))=S(x, u, w)+S(y, v, t) \\
\leq & S(x, x, a)+S(u, u, a)+S(w, w, a)+S(y, y, b) \\
\quad & +S(v, v, b)+S(t, t, b) \\
= & D_{s}((x, y),(x, y),(a, b)) \\
& +D_{s}((u, v),(u, v),(a, b)) \\
& +D_{s}((w, t),(w, t),(a, b)) .
\end{aligned}
$$

By the above, $D_{s}$ is an $S$-metric on $X \times X$.

Definition 9 ([16, Definition 1.5$])$. Let $(X, \preceq)$ be a partially ordered set and let $f, g: X \times X \mapsto X$ be two maps. We say the 
pair $(f, g)$ has the mixed weakly monotone property on $X$ if for all $x, y \in X$, we have

$$
\begin{aligned}
& x \leq f(x, y), f(y, x) \preceq y \\
& \text { implies } f(x, y) \\
& \leq g(f(x, y), f(y, x)), g(f(y, x), f(x, y)) \\
& \leq f(y, x), \\
& x \leq g(x, y), g(y, x) \preceq y \\
& \text { implies } g(x, y) \\
& \quad \preceq f(g(x, y), g(y, x)), f(g(y, x), g(x, y)) \\
& \quad \preceq g(y, x) .
\end{aligned}
$$

Example 10 ([16, Example 1.6]). Let $f, g: \mathbb{R} \times \mathbb{R} \mapsto \mathbb{R}$ be two functions given by

$$
\begin{aligned}
& f(x, y)=x-2 y, \\
& g(x, y)=x-y .
\end{aligned}
$$

Then the pair $(f, g)$ has the mixed weakly monotone property.

Definition 11 ([16, Definition 1.1$])$. Let $(X, \preceq)$ be a partially ordered set and let $f: X \times X \mapsto X$ be a map. We say the pair $f$ has the mixed monotone property on $X$ if for all $x, y \in X$, we have

$$
\begin{array}{r}
x_{1}, x_{2} \in X, x_{1} \preceq x_{2}, \\
\text { implies } f\left(x_{1}, y\right) \preceq f\left(x_{2}, y\right), \\
y_{1}, y_{2} \in X, y_{1} \preceq y_{2}, \\
\text { implies } f\left(x, y_{1}\right) \succeq f\left(x, y_{2}\right) .
\end{array}
$$

Remark 12 ([17, Remark 1.20]). Let $(X, \preceq)$ be a partially ordered set; let $f: X \times X \mapsto X$ be a map with the mixed monotone property on $X$. Then, for all $n \in \mathbb{N}$, the pair $\left(f^{n}, f^{n}\right)$ has the mixed weakly monotone property on $X$.

\section{Definition 13. An element $(x, y) \in X \times X$ is called a}

(1) coupled fixed point of a mapping $f: X \times X \mapsto X$ if $x=f(x, y)$ and $y=f(y, x)$;

(2) coupled common fixed point of two mappings $f, g$ : $X \times X \mapsto X$ if $x=f(x, y)=g(x, y)$ and $y=f(y, x)=$ $g(y, x)$.

\section{Main Results}

In this section, we establish some coupled common fixed point theorems by considering mappings on generalized metric spaces endowed with partial order. Before proceeding further, first, we define the following function which will be used in our results.

Let $\left\{x_{n}\right\}$ and $\left\{y_{n}\right\}$ be any two sequences of nonnegative real numbers. Define with $\Theta$ the set of all functions $\theta$ :
$[0, \infty)^{2} \mapsto[0,1)$ which, satisfying $\theta\left(x_{n}, y_{n}\right) \rightarrow 1$, implies $x_{n}, y_{n} \rightarrow 0$.

Some examples of such a function are as follows.

Example 14. Let $\theta:[0, \infty)^{2} \mapsto[0,1)$ be defined by

$$
\begin{aligned}
& \theta(x, y) \\
& = \begin{cases}\frac{\sin \left(k_{1} x+k_{2} y\right)}{k_{1} x+k_{2} y}, & x>0 \text { or } y>0, k_{1}, k_{2} \in(0,1) ; \\
l \in[0,1), & x=y=0 .\end{cases}
\end{aligned}
$$

Example 15. Let $\theta:[0, \infty)^{2} \mapsto[0,1)$ be defined by

$$
\begin{aligned}
& \theta(x, y) \\
& = \begin{cases}\frac{\ln \left(1+k_{1} x+k_{2} y\right)}{k_{1} x+k_{2} y}, & x>0 \text { or } y>0, k_{1}, k_{2} \in(0,1) ; \\
l \in[0,1), & x=y=0 .\end{cases}
\end{aligned}
$$

Example 16. Let $\theta:[0, \infty)^{2} \mapsto[0,1)$ be defined by

$$
\begin{aligned}
& \theta(x, y) \\
& = \begin{cases}\frac{\ln \left(1+\max \left\{k_{1} x, k_{2} y\right\}\right)}{\max \left\{k_{1} x, k_{2} y\right\}}, & x>0 \text { or } y>0, k_{1}, k_{2} \in(0,1) ; \\
l \in[0,1), & x=y=0 .\end{cases}
\end{aligned}
$$

Example 17. Let $\theta:[0, \infty)^{2} \mapsto[0,1)$ be defined by

$$
\begin{aligned}
& \theta(x, y) \\
& \quad= \begin{cases}1-k(x+y), & x>0 \text { or } y>0, k(x+y) \leq 1 ; \\
l \in[0,1), & k(x+y)>1 .\end{cases}
\end{aligned}
$$

Theorem 18. Let $(X, S)$ be a partially ordered S-metric space; let $f, g: X \times X \mapsto X$ be two maps such that

(1) $X$ is complete;

(2) the pair $(f, g)$ has the mixed weakly monotone property on $X$ :

$$
\begin{aligned}
x_{0} & \preceq f\left(x_{0}, y_{0}\right), \\
f\left(y_{0}, x_{0}\right) & \preceq y_{0} \\
\text { or } x_{0} & \preceq g\left(x_{0}, y_{0}\right), \\
g\left(y_{0}, x_{0}\right) & \preceq y_{0}
\end{aligned}
$$

for some $x_{0}, y_{0} \in X$;

(3) assume that there exists $\theta \in \Theta$ such that

$$
\begin{aligned}
& S(f(x, y), f(x, y), g(u, v)) \\
& +S(f(y, x), f(y, x), g(v, u)) \\
& \leq \theta(S(x, x, u), S(y, y, v))[S(x, x, u)+S(y, y, v)], \\
& \quad \text { for all } x, y, u, v \in X \text { with } x \leq u, y \geq v ;
\end{aligned}
$$


(4) $f$ or $g$ is continuous.

Then $f$ and $g$ have a coupled common fixed point in $X$.

Proof.

Step 1. We construct two Cauchy sequences in $X$.

Let $x_{0}, y_{0} \in X$ be such that $x_{0} \leq f\left(x_{0}, y_{0}\right), y_{0} \geq f\left(y_{0}, x_{0}\right)$.

Put $x_{1}=f\left(x_{0}, y_{0}\right), y_{1}=f\left(y_{0}, x_{0}\right), x_{2}=g\left(x_{1}, y_{1}\right), y_{2}=$ $g\left(y_{1}, x_{1}\right)$.

From the choice of $x_{0}, y_{0}$ and the fact that $(f, g)$ has mixed weakly monotone property we have

$$
\begin{aligned}
x_{1} & =f\left(x_{0}, y_{0}\right) \leq g\left(f\left(x_{0}, y_{0}\right), f\left(y_{0}, x_{0}\right)\right) \\
& =g\left(x_{1}, y_{1}\right)=x_{2} \Longrightarrow \\
x_{1} & \leq x_{2}, \\
x_{2} & =g\left(x_{1}, y_{1}\right) \leq f\left(g\left(x_{1}, y_{1}\right), g\left(y_{1}, x_{1}\right)\right) \\
& =f\left(x_{2}, y_{2}\right)=x_{3} \Longrightarrow \\
x_{2} & \leq x_{3} .
\end{aligned}
$$

Thus,

$$
\begin{aligned}
y_{1} & =f\left(y_{0}, x_{0}\right) \geq g\left(f\left(y_{0}, x_{0}\right), f\left(x_{0}, y_{0}\right)\right) \\
& =g\left(y_{1}, x_{1}\right)=y_{2} \Longrightarrow \\
y_{1} & \geq y_{2}, \\
y_{2} & =g\left(y_{1}, x_{1}\right) \geq f\left(g\left(y_{1}, x_{1}\right), g\left(x_{1}, y_{1}\right)\right) \\
& =f\left(y_{2}, x_{2}\right)=y_{3} \Longrightarrow
\end{aligned}
$$

$y_{2} \geq y_{3}$.

Continuing this way, we obtain

$$
\begin{aligned}
& x_{2 k+1}=f\left(x_{2 k}, y_{2 k}\right), \\
& y_{2 k+1}=f\left(y_{2 k}, x_{2 k}\right), \\
& x_{2 k+2}=g\left(x_{2 k+1}, y_{2 k+1}\right), \\
& y_{2 k+1}=g\left(y_{2 k+1}, x_{2 k+1}\right),
\end{aligned}
$$

for all $k \in \mathbb{N}$.

Therefore, the sequences $\left\{x_{n}\right\}$ and $\left\{y_{n}\right\}$ are monotone:

$$
\begin{aligned}
& x_{0} \leq x_{1} \leq \cdots \preceq x_{n} \preceq \cdots, \\
& y_{0} \geq y_{1} \geq \cdots \geq y_{n} \geq \cdots .
\end{aligned}
$$

Assume that there exists a nonnegative integer $n$ such that

$$
S\left(x_{n+1}, x_{n+1}, x_{n}\right)+S\left(y_{n+1}, y_{n+1}, y_{n}\right)=0 .
$$

It follows that

$$
S\left(x_{n+1}, x_{n+1}, x_{n}\right)=S\left(y_{n+1}, y_{n+1}, y_{n}\right)=0 .
$$

From the definition of $S$-metric space, we have $x_{n+1}=x_{n}$, $y_{n+1}=y_{n}$. It follows from (21) that $\left(x_{n}, y_{n}\right)$ is a coupled common fixed point of $f$ and $g$.

Now, we suppose that for all nonnegative $n$

$$
S\left(x_{n+1}, x_{n+1}, x_{n}\right)+S\left(y_{n+1}, y_{n+1}, y_{n}\right) \neq 0 .
$$

Using (18) and (21), for $n=2 k+1$, we have

$$
\begin{aligned}
& S\left(x_{2 k+1}, x_{2 k+1}, x_{2 k+2}\right)+S\left(y_{2 k+1}, y_{2 k+1}, y_{2 k+2}\right) \\
& \quad=S\left(f\left(x_{2 k}, y_{2 k}\right), f\left(x_{2 k}, y_{2 k}\right), g\left(x_{2 k+1}, y_{2 k+1}\right)\right) \\
& \quad+S\left(f\left(y_{2 k}, x_{2 k}\right), f\left(y_{2 k}, x_{2 k}\right), g\left(y_{2 k+1}, x_{2 k+1}\right)\right) \\
& \quad \leq \theta\left(S\left(x_{2 k}, x_{2 k}, x_{2 k+1}\right), S\left(y_{2 k}, y_{2 k}, y_{2 k+1}\right)\right) \\
& \cdot\left(S\left(x_{2 k}, x_{2 k}, x_{2 k+1}\right)+S\left(y_{2 k}, y_{2 k}, y_{2 k+1}\right)\right),
\end{aligned}
$$

which implies that

$$
\begin{gathered}
S\left(x_{2 k+1}, x_{2 k+1}, x_{2 k+2}\right)+S\left(y_{2 k+1}, y_{2 k+1}, y_{2 k+2}\right) \\
\quad<S\left(x_{2 k}, x_{2 k}, x_{2 k+1}\right)+S\left(y_{2 k}, y_{2 k}, y_{2 k+1}\right) .
\end{gathered}
$$

For all $k \in \mathbb{N}$, write

$$
\begin{aligned}
\gamma_{2 k+1}= & S\left(x_{2 k+1}, x_{2 k+1}, x_{2 k+2}\right) \\
& +S\left(y_{2 k+1}, y_{2 k+1}, y_{2 k+2}\right),
\end{aligned}
$$

and then the sequence $\left\{\gamma_{2 k+1}\right\}$ is monotone decreasing. Therefore, there exists $\gamma \geq 0$ such that

$$
\begin{aligned}
& \lim _{k \rightarrow \infty} \gamma_{2 k+1}=\lim _{k \rightarrow \infty}\left[S\left(x_{2 k+1}, x_{2 k+1}, x_{2 k+2}\right)\right. \\
& \left.+S\left(y_{2 k+1}, y_{2 k+1}, y_{2 k+2}\right)\right]=\gamma .
\end{aligned}
$$

We claim that $\gamma=0$. On the contrary, suppose that $\gamma>0$, and we have from (26) that

$$
\begin{aligned}
& \frac{S\left(x_{2 k+1}, x_{2 k+1}, x_{2 k+2}\right)+S\left(y_{2 k+1}, y_{2 k+1}, y_{2 k+2}\right)}{S\left(x_{2 k}, x_{2 k}, x_{2 k+1}\right)+S\left(y_{2 k}, y_{2 k}, y_{2 k+1}\right)} \\
& \quad \leq \theta\left(S\left(x_{2 k}, x_{2 k}, x_{2 k+1}\right), S\left(y_{2 k}, y_{2 k}, y_{2 k+1}\right)\right)<1 .
\end{aligned}
$$

Letting $k \rightarrow \infty$, we get

$$
\theta\left(S\left(x_{2 k}, x_{2 k}, x_{2 k+1}\right), S\left(y_{2 k}, y_{2 k}, y_{2 k+1}\right)\right) \longrightarrow 1 .
$$

Using the property of the function $\theta$, we have

$$
\begin{aligned}
S\left(x_{2 k}, x_{2 k}, x_{2 k+1}\right), S\left(y_{2 k}, y_{2 k}, y_{2 k+1}\right) \longrightarrow & \\
& \text { as } k \longrightarrow \infty .
\end{aligned}
$$

So, we have

$$
\begin{aligned}
S\left(x_{2 k}, x_{2 k}, x_{2 k+1}\right)+S\left(y_{2 k}, y_{2 k}, y_{2 k+1}\right) & \longrightarrow \\
& \text { as } k \longrightarrow \infty,
\end{aligned}
$$

which contradicts the assumption $\gamma>0$. Thus, $\gamma=0$. 
Analogously to $n=2 k+2$, we also have

$$
\begin{aligned}
& \lim _{k \rightarrow \infty}\left[S\left(x_{2 k+2}, x_{2 k+2}, x_{2 k+3}\right)+S\left(y_{2 k+2}, y_{2 k+2}, y_{2 k+3}\right)\right] \\
& \quad=0 .
\end{aligned}
$$

Thus, we have

$$
\lim _{n \rightarrow \infty}\left[S\left(x_{n}, x_{n}, x_{n+1}\right)+S\left(y_{n}, y_{n}, y_{n+1}\right)\right]=0 \text {. }
$$

Now, we have to prove that $\left\{x_{n}\right\}$ and $\left\{y_{n}\right\}$ are two Cauchy sequences in the $S$-metric space $(X, S)$. that

For all $n, m \in \mathbb{N}$ with $n \leq m$, by using Lemma 5 , we have

$$
\begin{aligned}
S & \left(x_{2 n+1}, x_{2 n+1}, x_{2 m+1}\right)+S\left(y_{2 n+1}, y_{2 n+1}, y_{2 m+1}\right) \\
& \leq\left(2 S\left(x_{2 n+1}, x_{2 n+1}, x_{2 n+2}\right)\right. \\
& \left.+2 S\left(y_{2 n+1}, y_{2 n+1}, y_{2 n+2}\right)\right) \\
& +\left(S\left(x_{2 n+2}, x_{2 n+2}, x_{2 m+1}\right)\right. \\
& \left.+S\left(y_{2 n+2}, y_{2 n+2}, y_{2 m+1}\right)\right) \\
& \leq\left(2 S\left(x_{2 n+1}, x_{2 n+1}, x_{2 n+2}\right)\right. \\
& \left.+2 S\left(y_{2 n+1}, y_{2 n+1}, y_{2 n+2}\right)\right) \\
& +\left(2 S\left(x_{2 n+2}, x_{2 n+2}, x_{2 n+3}\right)\right. \\
& \left.+2 S\left(y_{2 n+2}, y_{2 n+2}, y_{2 n+3}\right)\right)+\cdots \\
& +\left(2 S\left(x_{2 m-1}, x_{2 m-1}, x_{2 m}\right)\right. \\
& \left.+2 S\left(y_{2 m-1}, y_{2 m-1}, y_{2 m}\right)\right)+\left(S\left(x_{2 m}, x_{2 m}, x_{2 m+1}\right)\right. \\
+ & \left.S\left(y_{2 m}, y_{2 m}, y_{2 m+1}\right)\right)=2 \gamma_{2 n+1}+2 \gamma_{2 n+2}+\cdots \\
+ & 2 \gamma_{2 m-1}+\gamma_{2 m} .
\end{aligned}
$$

Taking the limit as $n, m \rightarrow \infty$ and using (35), we obtain

$$
S\left(x_{2 n+1}, x_{2 n+1}, x_{2 m+1}\right)+S\left(y_{2 n+1}, y_{2 n+1}, y_{2 m+1}\right) \longrightarrow 0 \text {. }
$$

Therefore,

$$
S\left(x_{2 n+1}, x_{2 n+1}, x_{2 m+1}\right), S\left(y_{2 n+1}, y_{2 n+1}, y_{2 m+1}\right) \longrightarrow 0 .
$$

By interchanging the roles of $f$ and $g$ and proceeding along the arguments discussed above, we also obtain that

$$
\begin{aligned}
S\left(x_{2 n}, x_{2 n}, x_{2 m+1}\right), S\left(y_{2 n}, y_{2 n}, y_{2 m+1}\right) & \longrightarrow 0, \\
S\left(x_{2 n}, x_{2 n}, x_{2 m}\right), S\left(y_{2 n}, y_{2 n}, y_{2 m}\right) & \longrightarrow 0, \\
S\left(x_{2 n+1}, x_{2 n+1}, x_{2 m}\right), S\left(y_{2 n+1}, y_{2 n+1}, y_{2 m}\right) & \longrightarrow 0 .
\end{aligned}
$$

Hence, for all $n, m \in \mathbb{N}$ with $n \leq m$, we get

$$
\lim _{n, m \rightarrow \infty}\left[S\left(x_{n}, x_{n}, x_{m}\right)+S\left(y_{n}, y_{n}, y_{m}\right)\right]=0 \text {. }
$$

It implies that

$$
\lim _{n, m \rightarrow \infty} S\left(x_{n}, x_{n}, x_{m}\right)=\lim _{n, m \rightarrow \infty} S\left(y_{n}, y_{n}, y_{m}\right)=0 .
$$

Therefore, $\left\{x_{n}\right\}$ and $\left\{y_{n}\right\}$ are two Cauchy sequences in the $S$ metric space $(X, S)$. Since $(X, S)$ is a complete $S$-metric space, hence $\left\{x_{n}\right\}$ and $\left\{y_{n}\right\}$ are $S$-convergent. Then there exist $x, y \in$ $X$ such that $x_{n} \rightarrow x$ and $y_{n} \rightarrow y$, respectively.

Step 2. We prove that $(x, y)$ is a coupled common fixed point of $f$ and $g$.

We consider the following two cases.

Case 1 ( $f$ is continuous). We have

$$
\begin{aligned}
x & =\lim _{n \rightarrow \infty} x_{n}=\lim _{n \rightarrow \infty} f\left(x_{n}, y_{n}\right) \\
& =f\left(\lim _{n \rightarrow \infty} x_{n}, \lim _{n \rightarrow \infty} y_{n}\right)=f(x, y), \\
y & =\lim _{n \rightarrow \infty} y_{n}=\lim _{n \rightarrow \infty} f\left(y_{n}, x_{n}\right) \\
& =f\left(\lim _{n \rightarrow \infty} y_{n}, \lim _{n \rightarrow \infty} x_{n}\right)=f(y, x) .
\end{aligned}
$$

Now using (18), we have

$$
\begin{aligned}
& S(f(x, y), f(x, y), g(x, y)) \\
& +S(f(y, x), f(y, x), g(y, x)) \\
& \leq \theta(S(x, x, x), S(y, y, y))[S(x, x, x)+S(y, y, y)] .
\end{aligned}
$$

That is,

$$
\begin{gathered}
S(x, x, g(x, y))+S(y, y, g(y, x)) \\
\leq \theta(S(x, x, x), S(y, y, y)) \\
\cdot[S(x, x, x)+S(y, y, y)] .
\end{gathered}
$$

Since $S(x, x, x)=S(y, y, y)=0$, we get $S(x, x, g(x, y))=$ $S(y, y, g(y, x))=0$; that is, $g(x, y)=x, g(y, x)=y$.

Therefore, $(x, y)$ is a coupled common fixed point of $f$ and $g$.

Case 2 ( $g$ is continuous). We also prove that $(x, y)$ is a coupled common fixed point of $f$ and $g$ similarly as in Case 1 .

Theorem 19. Let $(X, S)$ be a partially ordered S-metric space; let $f, g: X \times X \mapsto X$ be two maps such that

(1) $X$ is complete;

(2) the pair $(f, g)$ has the mixed weakly monotone property on $X$ :

$$
\begin{aligned}
x_{0} & \preceq f\left(x_{0}, y_{0}\right), \\
f\left(y_{0}, x_{0}\right) & \preceq y_{0} \\
\text { or } x_{0} & \preceq g\left(x_{0}, y_{0}\right), \\
g\left(y_{0}, x_{0}\right) & \preceq y_{0}
\end{aligned}
$$


(3) assume that there exists $\theta \in \Theta$ such that

$$
\begin{aligned}
& S(f(x, y), f(x, y), g(u, v)) \\
& +S(f(y, x), f(y, x), g(v, u)) \\
& \leq \theta(S(x, x, u), S(y, y, v))[S(x, x, u)+S(y, y, v)],
\end{aligned}
$$$$
\text { for all } x, y, u, v \in X \text { with } x \leq u, y \geq v ;
$$

(4) $X$ has the following properties:

(a) If $\left\{x_{n}\right\}$ is an increasing sequence with $x_{n} \rightarrow x$, then $x_{n} \preceq x$ for all $n \in \mathbb{N}$.

(b) If $\left\{x_{n}\right\}$ is a decreasing sequence with $x_{n} \rightarrow x$, then $x \preceq x_{n}$ for all $n \in \mathbb{N}$.

Then, $f$ and $g$ have a coupled common fixed point in $X$.

Proof. Proceeding along the same steps as in Theorem 18, we obtain a nondecreasing sequence $\left\{x_{n}\right\}$ converging to $x$ and a nonincreasing sequence $\left\{y_{n}\right\}$ converging to $y$, for some $x, y \in$ $X$. If $x_{n}=x$ and $y_{n}=y$ for all $n \geq 0$, then by construction, $x_{n+1}=x, y_{n+1}=y$. Thus, $(x, y)$ is a coupled common fixed point of $f$ and $g$. So we assume either $x_{n} \neq x$ or $y_{n} \neq y$ for $n \geq 0$. Then by using (18) and Lemma 5 , we have

$$
\begin{aligned}
S( & , x, f(x, y))+S(y, y, f(y, x)) \leq 2 S\left(x, x, x_{2 k+2}\right) \\
+ & S\left(x_{2 k+2}, x_{2 k+2}, f(x, y)\right)+2 S\left(y, y, y_{2 k+2}\right) \\
+ & S\left(y_{2 k+2}, y_{2 k+2}, f(y, x)\right)=2 S\left(x, x, x_{2 k+2}\right) \\
+ & S\left(g\left(x_{2 k+1}, y_{2 k+1}\right), g\left(x_{2 k+1}, y_{2 k+1}\right), f(x, y)\right) \\
+ & 2 S\left(y, y, y_{2 k+2}\right) \\
+ & S\left(g\left(y_{2 k+1}, x_{2 k+1}\right), g\left(y_{2 k+1}, x_{2 k+1}\right), f(y, x)\right) \\
& \leq 2 S\left(x, x, x_{2 k+2}\right)+2 S\left(y, y, y_{2 k+2}\right) \\
+ & \theta\left(S\left(x_{2 k+1}, x_{2 k+1}, x\right), S\left(y_{2 k+1}, y_{2 k+1}, y\right)\right) \\
+ & {\left[S\left(x_{2 k+1}, x_{2 k+1}, x\right)+S\left(y_{2 k+1}, y_{2 k+1}, y\right)\right] } \\
& <2 S\left(x, x, x_{2 k+2}\right)+2 S\left(y, y, y_{2 k+2}\right) \\
+ & S\left(x_{2 k+1}, x_{2 k+1}, x\right)+S\left(y_{2 k+1}, y_{2 k+1}, y\right) .
\end{aligned}
$$

Letting $n \rightarrow \infty$ in the above inequality, we get

$$
S(x, x, f(x, y))+S(y, y, f(y, x))=0 .
$$

Thus, $x=f(x, y), y=f(y, x)$. By interchanging the roles of $f$ and $g$ and using the same method mentioned above, we also get $x=g(x, y), y=g(y, x)$.

Hence, $(x, y)$ is a coupled common fixed point of $f$ and g.

Corollary 20. Let $(X, \preceq)$ be a partially ordered set and let $S$ be an $S$-metric on $X$ such that $(X, S)$ is a complete $S$-metric space. Suppose that $f, g: X \times X \mapsto X$ are two maps having the mixed weakly monotone property and assume that there exists $\mu \in \Theta$ such that

$$
\begin{aligned}
& S(f(x, y), f(x, y), g(u, v)) \leq \frac{1}{2} \\
& \cdot \mu(S(x, x, u), S(y, y, v))[S(x, x, u)+S(y, y, v)]
\end{aligned}
$$

for all $x, y, u, v \in X$ with $x \preceq u, y \geq v$.

Suppose that either

(1) $f$ or $g$ is continuous;

(2) X has the following property:

(a) If $\left\{x_{n}\right\}$ is an increasing sequence with $x_{n} \rightarrow x$, then $x_{n} \preceq x$ for all $n \in \mathbb{N}$.

(b) If $\left\{x_{n}\right\}$ is a decreasing sequence with $x_{n} \rightarrow x$, then $x \preceq x_{n}$ for all $n \in \mathbb{N}$.

If there exist $x_{0}, y_{0} \in X$ such that $x_{0} \preceq f\left(x_{0}, y_{0}\right), f\left(y_{0}, x_{0}\right) \preceq$ $y_{0}$ or $x_{0} \preceq g\left(x_{0}, y_{0}\right), g\left(y_{0}, x_{0}\right) \preceq y_{0}$, then $f$ and $g$ have $a$ coupled common fixed point in $X$.

Proof. For all $x, y, u, v \in X$, write

$$
\begin{gathered}
S(f(y, x), f(y, x), g(v, u)) \leq \frac{1}{2} \\
\cdot \mu(S(y, y, v), S(x, x, u)) \\
\cdot[S(y, y, v)+S(x, x, u)] .
\end{gathered}
$$

Adding (49) and (50), we get

$$
\begin{aligned}
& S(f(x, y), f(x, y), g(u, v)) \\
& \quad+S(f(y, x), f(y, x), g(v, u)) \\
& \quad \leq \frac{1}{2}[\mu(S(x, x, u), S(y, y, v)) \\
& \quad+\mu(S(y, y, v), S(x, x, u))][S(x, x, u) \\
& \quad+S(y, y, v)=\theta(S(x, x, u), S(y, y, v))[S(x, x, u) \\
& \quad+S(y, y, v)],
\end{aligned}
$$

where $\theta\left(\beta_{1}, \beta_{2}\right)=(1 / 2)\left[\mu\left(\beta_{1}, \beta_{2}\right)+\mu\left(\beta_{2}, \beta_{1}\right)\right]$, for all $\beta_{1}, \beta_{2} \in$ $[0, \infty)$.

It is easy to verify that $\theta \in \Theta$. Applying Theorems 18 and 19, we get desired result.

Remark 21. Taking $\mu\left(\beta_{1}, \beta_{2}\right)=k$ in Corollary 20 for all $\beta_{1}, \beta_{2} \in[0, \infty)$ and $k \in[0,1)$, we get the following corollary coinciding with [17, Corollary 2.4].

Corollary 22. In addition to the hypotheses of Corollary 20, suppose that for all $x, y, u, v \in X$ with $x \preceq u, y \geq v$, and some $k \in[0,1)$, inequality (49) in Corollary 20 is replaced by

$$
\begin{aligned}
& S(f(x, y), f(x, y), g(u, v)) \\
& \quad \leq \frac{k}{2}[S(x, x, u)+S(y, y, v)] .
\end{aligned}
$$

Then $f$ and $g$ have a coupled common fixed point in $X$. 
By choosing $f=g$ in Theorems 18 and 19 and using Remark 12, we get coupled fixed point theorem of $f$ written by the following corollary.

Corollary 23. Let $(X, \preceq)$ be a partially ordered S-metric space and let $f: X \times X \mapsto X$ be a map such that

(1) $X$ is complete;

(2) $f$ has the mixed monotone property on $X$;

$$
\begin{aligned}
x_{0} & \leq f\left(x_{0}, y_{0}\right), \\
f\left(y_{0}, x_{0}\right) & \leq y_{0}
\end{aligned}
$$

$$
\text { for some } x_{0}, y_{0} \in X
$$

(3) assume that there exists $\theta \in \Theta$ such that

$$
\begin{aligned}
& S(f(x, y), f(x, y), f(u, v)) \\
& +S(f(y, x), f(y, x), f(v, u)) \\
& \leq \theta(S(x, x, u), S(y, y, v))[S(x, x, u)+S(y, y, v)], \\
& \quad \text { for all } x, y, u, v \in X \text { with } x \leq u, y \geq v ;
\end{aligned}
$$

(4) $f$ is continuous or $X$ has the following properties:

(a) If $\left\{x_{n}\right\}$ is an increasing sequence with $x_{n} \rightarrow x$, then $x_{n} \preceq x$ for all $n \in \mathbb{N}$.

(b) If $\left\{x_{n}\right\}$ is a decreasing sequence with $x_{n} \rightarrow x$, then $x \leq x_{n}$ for all $n \in \mathbb{N}$.

Then $f$ has a coupled common fixed point in $X$.

Theorem 24. In addition to the hypotheses of Theorem 18, suppose that, for all $(x, y),(s, t) \in X \times X$, there exists $(p, q) \in$ $X \times X$ that is comparable with $(x, y)$ and $(s, t)$. Then $f$ and $g$ have a unique coupled fixed point in $X$.

Proof. By Theorem 18, $f$ and $g$ have a coupled common fixed point $(x, y)$. Let $(s, t)$ be another coupled common fixed point of $f$ and $g$.

By assumption, there exists $(p, q) \in X \times X$ that is comparable to $(x, y)$ and $(s, t)$. Put $p=p_{0}, q=q_{0}$ and choose $p_{1}, q_{1} \in X$ such that $p_{1}=f\left(p_{0}, q_{0}\right), q_{1}=f\left(q_{0}, p_{0}\right)$. Using the same construction as in the proof of Theorem 18, we have two sequences $\left\{p_{n}\right\}$ and $\left\{q_{n}\right\}$ such that

$$
\begin{aligned}
& p_{2 k+1}=f\left(p_{2 k}, q_{2 k}\right), \\
& q_{2 k+1}=f\left(q_{2 k}, p_{2 k}\right), \\
& p_{2 k+2}=g\left(p_{2 k+1}, q_{2 k+1}\right), \\
& q_{2 k+2}=g\left(q_{2 k+1}, p_{2 k+1}\right),
\end{aligned}
$$

satisfying

$$
\begin{aligned}
& p_{0} \preceq p_{1} \preceq \cdots \preceq p_{n} \preceq \cdots, \\
& q_{0} \succeq q_{1} \succeq \cdots \succeq q_{n} \geq \cdots .
\end{aligned}
$$

Since $(p, q)$ is comparable to $(x, y)$, we can assume that $(x, y) \geq(p, q)=\left(p_{0}, q_{0}\right)$.

Then it is easy to show that $\left(p_{n}, q_{n}\right)$ and $(x, y)$ are comparable; that is, $(x, y) \geq\left(p_{n}, q_{n}\right)$, for all $n \in \mathbb{N}$.

For $n=2 k+1$, from (18) we have

$$
\begin{aligned}
S & \left(p_{2 k+1}, x, x\right)+S\left(q_{2 k+1}, y, y\right) \\
& =S\left(f\left(p_{2 k}, q_{2 k}\right), f(x, y), f(x, y)\right) \\
& +S\left(f\left(q_{2 k}, p_{2 k}\right), f(y, x), f(y, x)\right) \\
& \leq \theta\left(S\left(p_{2 k}, x, x\right), S\left(q_{2 k}, y, y\right)\right) \\
& \cdot\left[S\left(p_{2 k}, x, x\right)+S\left(q_{2 k}, y, y\right)\right]
\end{aligned}
$$

which implies

$$
\begin{aligned}
& S\left(p_{2 k+1}, x, x\right)+S\left(q_{2 k+1}, y, y\right) \\
& \quad<S\left(p_{2 k}, x, x\right)+S\left(q_{2 k}, y, y\right) .
\end{aligned}
$$

We see that the sequence $\left\{S\left(p_{2 k}, x, x\right)+S\left(q_{2 k}, y, y\right)\right\}$ is decreasing, and there exist $\xi \geq 0$ such that

$$
S\left(p_{2 k}, x, x\right)+S\left(q_{2 k}, y, y\right) \longrightarrow \xi, \quad \text { as } k \longrightarrow \infty .
$$

Now, we have to show that $\xi=0$. On the contrary, suppose that $\xi>0$. Following the same arguments as in the proof of Theorem 18, we obtain

$$
\theta\left(S\left(p_{2 k}, x, x\right), S\left(q_{2 k}, y, y\right)\right) \longrightarrow 1, \text { as } k \longrightarrow \infty .
$$

It follows that

$$
S\left(p_{2 k}, x, x\right), S\left(q_{2 k}, y, y\right) \longrightarrow 0, \quad \text { as } k \longrightarrow \infty .
$$

This implies

$$
S\left(p_{2 k}, x, x\right)+S\left(q_{2 k}, y, y\right) \longrightarrow 0, \quad \text { as } k \longrightarrow \infty,
$$

which is not possible in virtue of (30). Hence, $\xi=0$. Therefore, (59) becomes

$$
S\left(p_{2 k}, x, x\right)+S\left(q_{2 k}, y, y\right) \longrightarrow 0, \quad \text { as } k \longrightarrow \infty .
$$

Similarly, we can get that

$$
S\left(p_{2 k}, s, s\right)+S\left(q_{2 k}, t, t\right) \longrightarrow 0, \quad \text { as } k \longrightarrow \infty .
$$

Using (63)-(64), the second condition of $S$-metric, and taking the limit $k \rightarrow \infty$, we obtain that

$$
S(s, x, x)+S(t, y, y)=0 .
$$

Thus, we conclude that $x=s, y=t$.

Analogous to $n=2 k+1$, by interchanging the roles of $f$ and $g$, (65) holds true for $n=2 k+2$.

Therefore, we conclude that $f$ and $g$ have a unique coupled common fixed point.

Similarly, we can prove the following theorem. 
Theorem 25. In addition to the hypotheses of Theorem 19, suppose that, for all $(x, y),(s, t) \in X \times X$, there exists $(p, q) \in$ $X \times X$ that is comparable with $(x, y)$ and $(s, t)$. Then $f$ and $g$ have a unique coupled fixed point in $X$.

Finally, we give some examples to demonstrate the validity of our results.

Example 26. Let $X=[0, \pi / 4)$, with the $S$-metric defined by $S(x, y, z)=|x-z|+|y-z|$ and the natural ordering of real numbers $\leq$. Then $X$ is a totally ordered, complete $S$-metric space.

Let $\theta:[0, \pi / 4)^{2} \mapsto[0,1)$ be defined by

$$
\theta(x, y)= \begin{cases}1-\frac{\sin (x+y)}{2} ; & x+y>0 \\ 0 ; & x=y=0 .\end{cases}
$$

For all $x, y \in X$, put $f(x, y)=g(x, y)=(2 x-y+15) / 16$.

The pair $(f, g)$ has the mixed weakly monotone property and $\forall x, y, u, v \in X$ with $x \leq u, y \geq v$, we have that

$$
\begin{aligned}
S( & f(x, y), f(x, y), g(u, v)) \\
+ & S(f(y, x), f(y, x), g(v, u)) \\
& =2|f(x, y)-g(u, v)|+2|f(y, x)-g(v, u)| \\
& =2\left|\frac{2 x-y+15}{16}-\frac{2 u-v+15}{16}\right| \\
& +2\left|\frac{2 y-x+15}{16}-\frac{2 v-u+15}{16}\right| \\
& =\frac{1}{8}|2(x-u)+(v-y)|+\frac{1}{8}|2(y-v)+(u-x)| \\
& \leq \frac{1}{8}(2|x-u|+|v-y|) \\
& +\frac{1}{8}(|2(y-v)|+|u-x|) \leq \frac{1}{2}(|x-u|+|v-y|) \\
& \leq\left(1-\frac{\sin (|x-u|+|v-y|)}{2}\right)(|x-u|+|v-y|) \\
& =\theta(S(x, x, u), S(y, y, v)) \\
& {[S(x, x, u)+S(y, y, v)] . }
\end{aligned}
$$

Then the contractive condition (18) in Theorem 18 holds, and $(1,1)$ is the unique coupled common fixed point.

Example 27. Let $X=[0,1]$, with the $S$-metric defined by $S(x, y, z)=(1 / 2)(|x-z|+|y-z|)$ and the natural ordering of real numbers $\leq$. Then $X$ is a totally ordered, complete $S$ metric space.

Let $\theta:[0, \infty)^{2} \mapsto[0,1)$ be defined by

$$
\theta(x, y)= \begin{cases}\frac{\ln (1+\max \{x, y\})}{\max \{x, y\}}, & x>0 \text { or } y>0 ; \\ l \in[0,1), & x=y=0 .\end{cases}
$$

For all $x, y \in X$, put $f(x, y)=g(x, y)=(1 / 8)[\ln (1+x)-$ $2 \ln (1+y)]$.
The pair $(f, g)$ has the mixed weakly monotone property. $\forall x, y, u, v \in X$ with $x \leq u, y \geq v$, we have that

$S(f(x, y), f(x, y), g(u, v))$

$+S(f(y, x), f(y, x), g(v, u))$

$=\mid \frac{\ln (1+x)-2 \ln (1+y)}{8}$

$-\frac{\ln (1+u)-2 \ln (1+v)}{8}$

$+\mid \frac{\ln (1+y)-2 \ln (1+x)}{8}$

$-\frac{\ln (1+v)-2 \ln (1+u)}{8}$

$=\frac{1}{8} \mid(\ln (1+x)-\ln (1+u))$

$+2(\ln (1+v)-\ln (1+y)) \mid$

$+\frac{1}{8} \mid(\ln (1+y)-\ln (1+v))$

$+2(\ln (1+u)-\ln (1+x))\left|\leq \frac{1}{8}\right| \ln (1+x)$

$-\ln (1+u)\left|+\frac{1}{4}\right| \ln (1+v)-\ln (1+y) \mid$

$+\frac{1}{8}|\ln (1+y)-\ln (1+v)|+\frac{1}{4} \mid \ln (1+u)$

$-\ln (1+x)\left|\leq \frac{1}{2}\right| \ln (1+u)-\ln (1+x) \mid$

$+\frac{1}{2}|\ln (1+y)-\ln (1+v)|=\frac{1}{2}\left|\ln \left(\frac{1+u}{1+x}\right)\right|$

$+\frac{1}{2}\left|\ln \left(\frac{1+y}{1+v}\right)\right|=\frac{1}{2}\left|\ln \left(1+\frac{u-x}{1+x}\right)\right|$

$+\frac{1}{2}\left|\ln \left(1+\frac{y-v}{1+v}\right)\right| \leq \frac{1}{2}|\ln (1+(u-x))|$

$+\frac{1}{2}|\ln (1+(y-v))|=\frac{1}{2} \ln (1+|u-x|)+\frac{1}{2}$

$\cdot \ln (1+|y-v|) \leq \ln (1+\max \{|x-u|,|y-v|\})$

$=\frac{\ln (1+\max \{|x-u|,|y-v|\})}{\max \{|x-u|,|y-v|\}}$

$\cdot \max \{|x-u|,|y-v|\}$

$\leq \frac{\ln (1+\max \{|x-u|,|y-v|\})}{\max \{|x-u|,|y-v|\}}(|x-u|$

$+|y-v|)=\theta(S(x, x, u), S(y, y, v))[S(x, x, u)$

$+S(y, y, v)]$. 
Then the contractive condition (18) in Theorem 18 holds, and $(0,0)$ is the unique coupled common fixed point.

\section{Conflict of Interests}

The authors declare that they have no competing interests.

\section{Authors' Contribution}

Both authors contributed equally and significantly to writing this paper. All authors read and approved the final paper.

\section{Acknowledgments}

The authors thank Dr. Dolićanin-Djekic Diana (Faculty of Technical Science, University of Pristina-Kosovska Mitrovica, Serbia) for her editing and polishing work for the revised paper. This work is partially supported by Natural Science Foundation of China (Grant no. 61573010), Natural Science Foundation of Hainan Province (Grant no. 114014), Opening Project of Sichuan Province University Key Laboratory of Bridge Non-Destruction Detecting and Engineering Computing (2015QZJ01), Artificial Intelligence of Key Laboratory of Sichuan Province (2015RZJ01), Scientific Research Fund of Sichuan Provincial Education Department (14ZB0208 and 16ZA0256), and Scientific Research Fund of Sichuan University of Science and Engineering (2014RC01 and 2014RC03).

\section{References}

[1] S. Banach, "Sur les opérations dans les ensembles abstraits et leur application aux équations intégrals," Fundamenta Mathematicae, vol. 3, pp. 133-181, 1922.

[2] M. A. Geraghty, "On contractive mappings," Proceedings of the American Mathematical Society, vol. 40, pp. 604-608, 1973.

[3] A. Amini-Harandi and H. Emami, "A fixed point theorem for contraction type maps in partially ordered metric spaces and application to ordinary differential equations," Nonlinear Analysis: Theory, Methods \& Applications, vol. 72, no. 5, pp. 2238-2242, 2010.

[4] C. Mongkolkeha, Y. J. Cho, and P. Kumam, "Best proximity points for Geraghty's proximal contraction mappings," Fixed Point Theory and Applications, vol. 2013, article 180, 2013.

[5] V. La Rosa and P. Vetro, "Fixed points for Geraghty-contractions in partial metric spaces," Journal of Nonlinear Science and Its Applications, vol. 7, no. 1, pp. 1-10, 2014.

[6] Z. Kadelburg, P. Kumam, S. Radenović, and S. Sintunavarat, "Common coupled fixed point theorems for Geraghty-type contraction mappings using monotone property," Fixed Point Theory and Applications, vol. 2015, article 27, 2015.

[7] Z. Kadelburg, S. Radenović, and M. Rajović, "A note on fixed point theorems for rational Geraghty contractive mappings in ordered b-metric spaces," Kragujevac Journal of Mathematics, vol. 39, no. 2, pp. 187-195, 2015.

[8] H. Huang, S. Radenović, and J. Vujaković, "On some recent coincidence and immediate consequences in partially ordered b-metric spaces," Fixed Point Theory and Applications, vol. 2015, article 63, 18 pages, 2015.
[9] T. V. An, N. V. Dung, Z. Kadelburg, and S. Radenović, "Various generalizations of metric spaces and fixed point theorems," Revista de la Real Academia de Ciencias Exactas, Fisicas y Naturales, Serie A. Matematicas, vol. 109, no. 1, pp. 175-198, 2015.

[10] Z. M. Fadail, A. G. Ahmad, G. S. Rad, V. Ozturk, and S. Radenović, "Some remarks on coupled, tripled and n-tupled fixed points theorems in ordered abstract metric spaces," Far East Journal of Mathematical Sciences, vol. 97, no. 7, pp. 809-839, 2015.

[11] H.-S. Ding, M. Imdad, S. Radenović, and J. Vujaković, "On some fixed point results in b-metric, rectangular and b-rectangular metric spaces," Arab Journal of Mathematical Sciences, 2015.

[12] M. Abbas, I. Zulfaqar, and S. Radenović, "Common fixed point of $(\psi, \beta)$-generalized contractive mappings in partially ordered metric spaces," Chinese Journal of Mathematics, vol. 2014, Article ID 379049, 9 pages, 2014.

[13] Z. Kadelburg, M. Pavlović, and S. Radenović, "Common fixed point theorems for ordered contractions and quasicontractions in ordered cone metric spaces," Computers \& Mathematics with Applications, vol. 59, no. 9, pp. 3148-3159, 2010.

[14] S. Sedghi, N. Shobe, and A. Aliouche, "A generalization of fixed point theorem in S-metric spaces," Matematički Vesnik, vol. 64, no. 3, pp. 258-266, 2012.

[15] S. Sedghi and N. V. Dung, "Fixed point theorems on S-metric spaces," Matematichki Vesnik, vol. 66, no. 1, pp. 113-124, 2014.

[16] M. E. Gordji, E. Akbartabar, Y. J. Cho, and M. Ramezani, "Coupled common fixed point theorems for mixed weakly monotone mappings in partially ordered metric spaces," Fixed Point Theory and Applications, vol. 2012, article 95, 2012.

[17] N. V. Dung, "On coupled common fixed points for mixed weakly monotone maps in partially ordered $S$-metric spaces," Fixed Point Theory and Applications, vol. 2013, article 48, 17 pages, 2013.

[18] T. Gnana Bhaskar and V. Lakshmikantham, "Fixed point theorems in partially ordered metric spaces and applications," Nonlinear Analysis: Theory, Methods \& Applications, vol. 65, no. 7, pp. 1379-1393, 2006. 


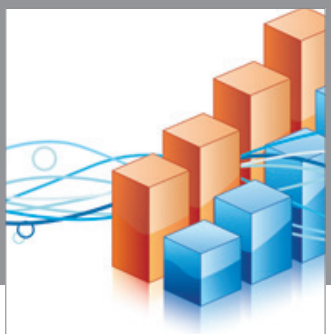

Advances in

Operations Research

vatem alat4

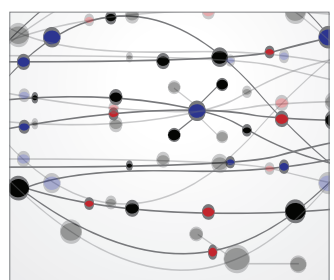

\section{The Scientific} World Journal
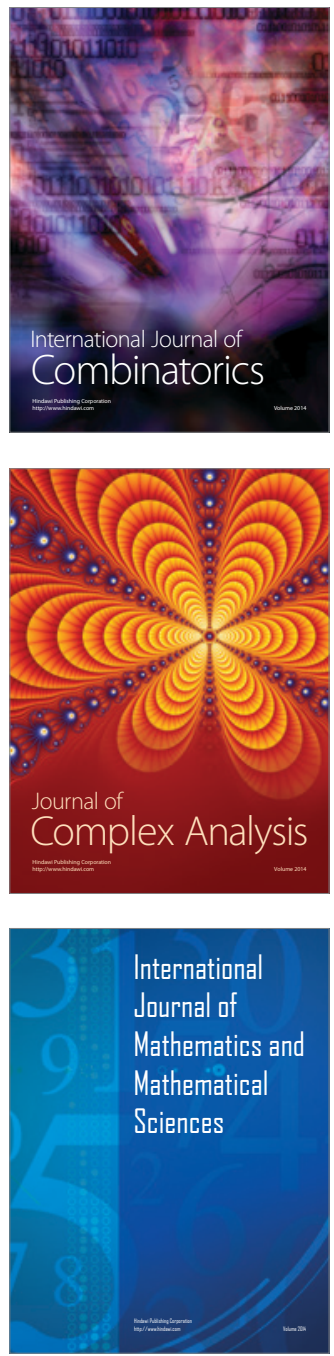
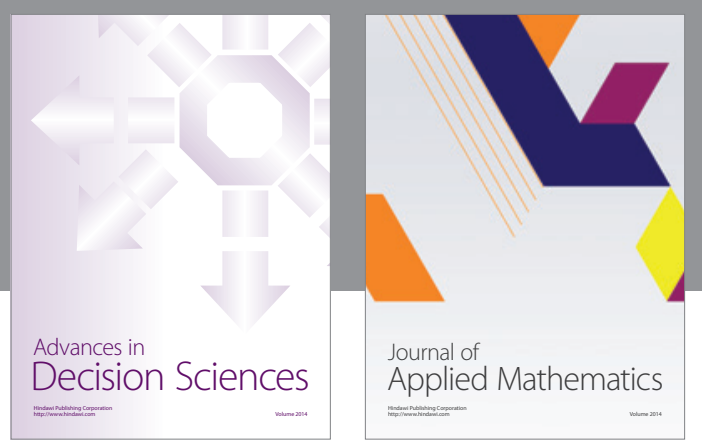

Algebra

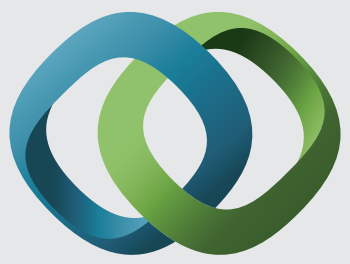

\section{Hindawi}

Submit your manuscripts at

http://www.hindawi.com
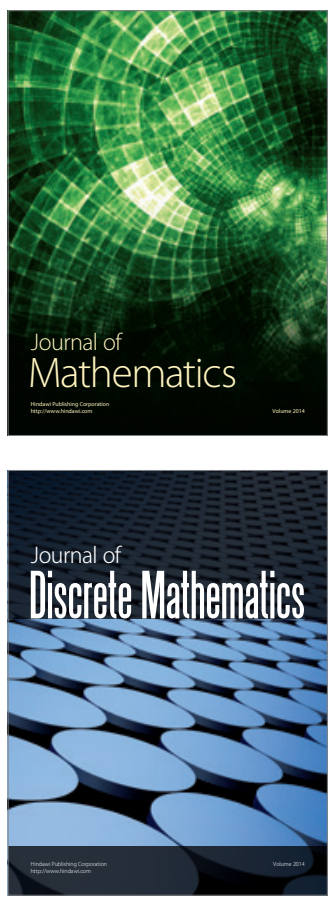

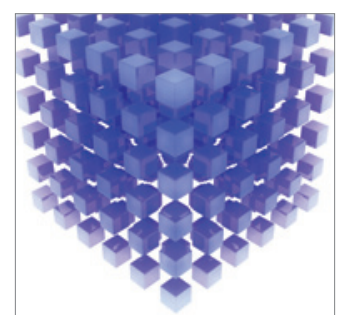

Mathematical Problems in Engineering
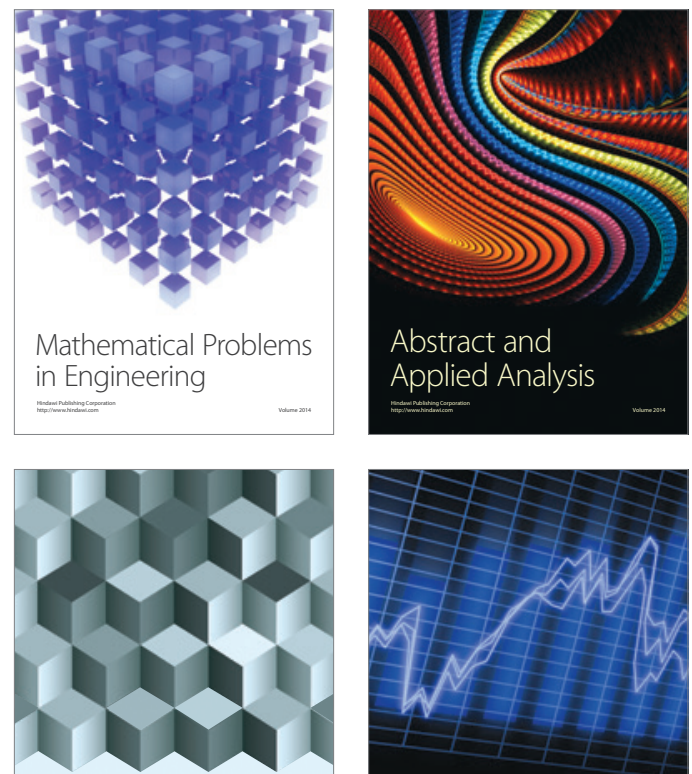

Journal of

Function Spaces

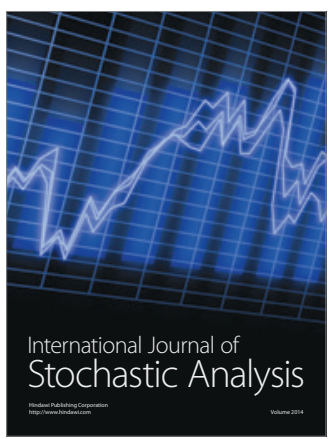

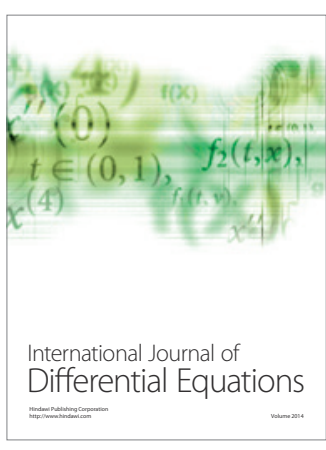
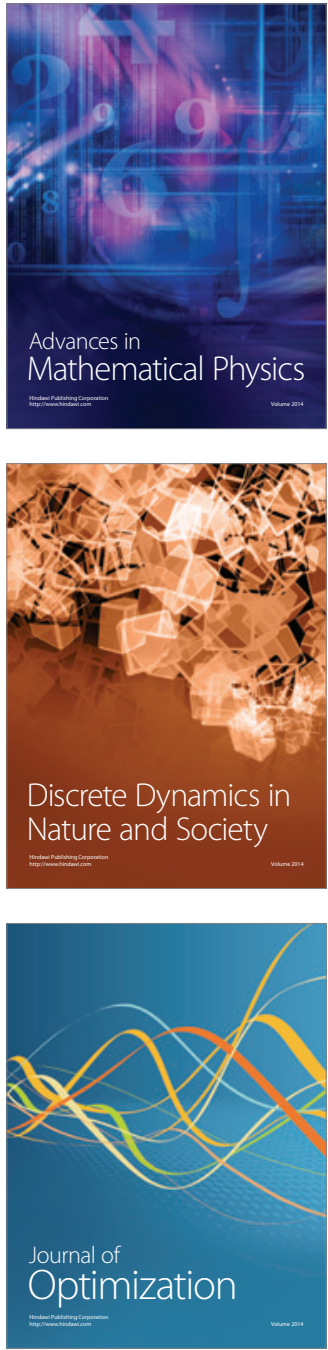